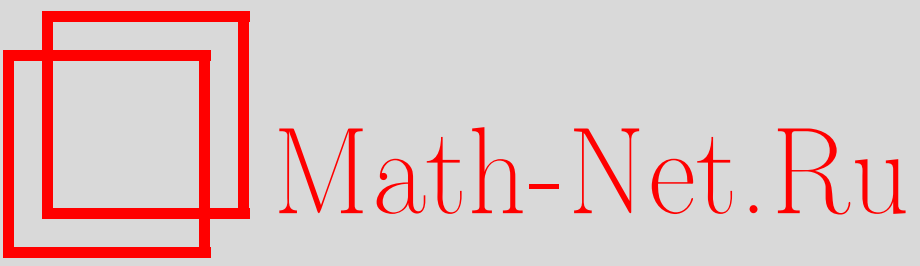

О. А. Матевосян, О единственности решений задачи Робэна для системы теории упругости в полупространстве, УМН, 2003, том 58, выпуск 4, 151-152

DOI: https://doi.org/10.4213/rm650

Использование Общероссийского математического портала Math-Net.Ru подразумевает, что вы прочитали и согласны с пользовательским соглашением

http://www.mathnet.ru/rus/agreement

Параметры загрузки:

IP: 54.84 .234 .179

26 апреля 2023 г., 13:07:43 


\title{
О ЕДИНСТВЕННОСТИ РЕШЕНИЙ ЗАДАЧИ РОБЭНА ДЛЯ СИСТЕМЫ ТЕОРИИ УПРУГОСТИ В ПОЛУПРОСТРАНСТВЕ
}

\author{
О. А. МАТЕВосян
}

Пусть $x=\left(x_{1}, \ldots, x_{n}\right) \in \mathbb{R}^{n}, n \geqslant 2$. Положим $x=\left(x^{\prime}, x_{n}\right)$, где $x^{\prime}=\left(x_{1}, \ldots, x_{n-1}\right) \in$ $\mathbb{R}^{n-1} ;$ тогда $\mathbb{R}_{+}^{n}=\left\{x=\left(x^{\prime}, x_{n}\right) \in \mathbb{R}^{n}: x_{n}>0\right\}$ - "открытое полупространство" с границей $\partial \mathbb{R}_{+}^{n}:=\left\{x=\left(x^{\prime}, x_{n}\right) \in \mathbb{R}^{n}: x_{n}=0\right\} \equiv \mathbb{R}^{n-1},|x|=\sqrt{x_{1}^{2}+\cdots+x_{n}^{2}}$.

Рассмотрим стационарную линейную систему теории упругости

$$
L u \equiv(L u)_{i}=\sum_{j, k, h=1}^{n} \frac{\partial}{\partial x_{k}}\left(a_{k h}^{i j} \frac{\partial u_{j}(x)}{\partial x_{h}}\right)=0, \quad i=1, \ldots, n,
$$

в области $\Omega \equiv \mathbb{R}_{+}^{n}=\left\{x=\left(x^{\prime}, x_{n}\right) \in \mathbb{R}^{n}: x_{n}>0\right\}$ с границей $\partial \Omega:=\left\{x=\left(x^{\prime}, x_{n}\right) \in \mathbb{R}^{n}\right.$ : $\left.x_{n}=0\right\}, u=\left(u_{1}, \ldots, u_{n}\right)$. Здесь и далее суммирование проводится по повторяющимся индексам от 1 до $n$. Предполагается, что коэффициенты постоянные и выполнены условия: $a_{k h}^{i j}=a_{h k}^{j i}=$ $a_{i h}^{k j}, \lambda_{1}|\xi|^{2} \leqslant a_{k h}^{i j} \xi_{k}^{i} \xi_{h}^{j} \leqslant \lambda_{2}|\xi|^{2}$, где $\xi$ - произвольная симметрическая матрица $\left\{\xi_{k}^{i}\right\}, \xi_{k}^{i}=\xi_{i}^{k}$, $|\xi|^{2}=\xi_{k}^{i} \xi_{k}^{i}, \lambda_{1}, \lambda_{2}$-положительные постоянные.

Для системы (1) будем рассматривать задачу Робэна: найти вектор-функцию $u(x)$, удовлетворяющую системе (1) в области $\Omega$ и однородному граничному условию

$$
\sigma(u)+\tau(x) u(x)=0 \text { на } \partial \Omega,
$$

где $\sigma(u)=\left(\sigma_{1}(u), \ldots, \sigma_{n}(u)\right), \sigma_{i}(u) \equiv a_{k h}^{i j} \frac{\partial u_{j}(x)}{\partial x_{h}} \nu_{k}, i=1, \ldots, n, \nu=\left(\nu_{1}, \ldots, \nu_{n}\right)$ - единичный вектор внешней нормали к $\partial \Omega, \tau(x)$ - бесконечно гладкая функция с равномерно ограниченными производными на $\partial \Omega, \tau \geqslant 0, \tau \not \equiv 0$.

Положим $D^{\alpha} u=\partial^{|\alpha|} u / \partial x_{1}^{\alpha_{1}} \cdots \partial x_{n}^{\alpha_{n}}$, где $\alpha=\left(\alpha_{1}, \ldots, \alpha_{n}\right), \alpha_{i} \geqslant 0$ - целше числа, $|\alpha|=$ $\alpha_{1}+\cdots+\alpha_{n}$. Пусть $\Omega_{R}=\Omega \cap\left\{x \in \mathbb{R}^{n}:|x|<R\right\}$,

$$
D(u(x), \Omega)=\int_{\Omega} \sum_{i, j=1}^{n}\left(\frac{\partial u_{i}}{\partial x_{j}}\right)^{2} d x, \quad E(u(x), \Omega)=\int_{\Omega} \sum_{i, j=1}^{n}\left(\frac{\partial u_{i}}{\partial x_{j}}+\frac{\partial u_{j}}{\partial x_{i}}\right)^{2} d x .
$$

Краевые задачи для системы теории упругости в ограниченных областях хорошо изучены в монографиии Г. Фикеры [1]. В работах В. А. Кондратьева и О. А. Олейник [2], [3] для ограниченных и широкого класса неограниченных областей установлены обобщения неравенств Корна и Харди, с помощью которых исследованы основные краевые задачи для системы теории упругости. Как известно, в случае, когда $\Omega$ - неограниченная область, следует дополнительно охарактеризовать поведение решения на бесконечности. Как правило, для этой цели служит либо условие конечности интеграла Дирихле $D(u(x), \Omega)$ или интеграла энергии $E(u(x), \Omega)$, либо условие на характер убьвания модуля решения при $|x| \rightarrow \infty$.

В заметке изучаются свойства обобщенных решений задачи Робэна для системы теории упругости в полупространстве с условием конечности весового интеграла энергии:

$$
E_{a}(u(x), \Omega) \equiv \int_{\Omega}|x|^{a} \sum_{i, j=1}^{n}\left(\frac{\partial u_{i}}{\partial x_{j}}+\frac{\partial u_{j}}{\partial x_{i}}\right)^{2} d x<\infty, \quad a \in \mathbb{R}^{1} .
$$

При том же условии на поведение решения на бесконечности в разных классах неограниченных областей автором [4], [5] изучены вопросы единственности и найдены размерности пространства решений краевых задач для системы теории упругости и бигармонического уравнения.

ОПРедЕлЕниЕ. Обобщенным решением задачи Робәна для системы (1) в $\Omega$ с граничньм условием (2) на $\partial \Omega$ будем назьвать вектор-функцию $u(x) \in \stackrel{\circ}{H_{\text {loc }}^{1}}(\Omega)$ такую, что для всякой вектор-функции $\psi(x) \in H^{1}(\Omega)$ выполнено интегральное тождество

$$
\int_{\Omega} a_{k h}^{i j} \frac{\partial u_{j}(x)}{\partial x_{h}} \frac{\partial \psi_{i}(x)}{\partial x_{k}} d x+\int_{\partial \Omega} \tau(x) u_{i}(x) \psi_{i}(x) d s=0 .
$$


Лемма. Пусть и $(x)$ - решение системы (1) в $\Omega$, удовлетворяющее граничному условию (2) и неравенству $D\left(u(x), \Omega_{R}\right) \leqslant C\left(1+R^{m}\right)$, где $C$ - некоторая положительная постоянная, $m \geqslant 0$ - целое число. Тогда и(x) - многочлен степени не выше $m$, т.е. $u(x)=P(x)$, ord $P(x) \leqslant m$.

ДокАЗАТЕльСтво. Эта лемма является некоторым аналогом теоремы Лиувилля для системы уравнений и справедлива для общих эллиптических систем по Дуглису-Ниренбергу с постоянными коэффициентами. В случае, когда $\Omega \equiv \mathbb{R}^{n}$, она доказана в [6].

Доказательство при $\Omega \equiv \mathbb{R}_{+}^{n}$ аналогично. Именно в теории эллиптических систем установлено неравенство Бернштейна:

$$
\int_{|x| \leqslant 1 / 2} \sum_{|\alpha|=l}\left|D^{\alpha} u(x)\right|^{2} d x \leqslant C_{1} \int_{|x| \leqslant 1}|u(x)|^{2} d x, \quad l \geqslant 1,
$$

где постоянная $C_{1}$ не зависит от $u$.

Если $u(x)$ является решением однородной эллиптической системы с постоянными коэффициентами в полушаре $|x| \leqslant 1, x_{n}>0$ и при $x_{n}=0$ удовлетворяет нулевым краевым условиям Шапиро-Лопатинского [7] (более подробно см. [8]), то, сделав в (3) замену $x=\lambda y$, получим в силу однородности систем и краевых условий

$$
\begin{aligned}
I(\lambda) & =\int_{|x| \leqslant \lambda / 2} \sum_{|\alpha|=l}\left|D^{\alpha} u(x)\right|^{2} d x \leqslant \lambda^{-l} \int_{|x| \leqslant \lambda}|u(x)|^{2} d x \\
& \leqslant 2 \lambda^{-l}\left(\int_{|x| \leqslant \lambda}|u(x)-C|^{2} d x+\int_{|x| \leqslant \lambda} C^{2} d x\right) \\
& \leqslant 2 \lambda^{-l}\left(C \lambda^{2} \int_{|x| \leqslant \lambda}|\nabla u(x)|^{2} d x+C^{2} \lambda^{n}\right),
\end{aligned}
$$

из условий леммы имеем $I(\lambda) \leqslant 2 \lambda^{-l}\left(C \lambda^{2} \lambda^{m}+C^{2} \lambda^{n}\right)$.

Взяв $l>\max \{2+m, n\}$ и устремив $\lambda \rightarrow \infty$, получим $D^{\alpha} u=0$ при $|x| \leqslant 1$. Следовательно, $u(x)$ является многочленом степени меньше $l$ при $|x| \leqslant 1$. Из условия $\left|D\left(u(x), \Omega_{R}\right)\right| \leqslant C\left(1+R^{m}\right)$ вытекает, что степень такого многочлена не вьше $m$. Лемма доказана.

Теорема 1. Пусть и $(x)$ является обобщенным решением задачи Робэна для системы (1) в $\Omega$ с граничным условием (2) на $\partial \Omega$ и с условием $E(u(x), \Omega)<\infty$. Тогда $u(x)=0$.

Теорема 2. Задача Робэна для системы (1) в $\Omega$ с граничным условием (2) на $2 \Omega и$ с условием $E_{a}(u(x), \Omega)<\infty$, имеет:

(i) только тривиальное решение при $-n \leqslant a<\infty$;

(ii) $n$ линейно независимых решений $п р и-2-n \leqslant a<-n$.

Теорема 3. Задача Робэна для системы (1) в $\Omega$ с граничным условием (2) на д $и$ с условием $E_{a}(u(x), \Omega)<\infty$ имеет $k(r, n)$ линейно независимых решений при $-2 r-n \leqslant$ $a<-2 r-n+2, r \geqslant 2$, əде

$$
k(r, n)=n\left(\frac{(r+n) !}{n ! r !}-\frac{(r+n-2) !}{n !(r-2) !}-\frac{(r+n-1) !}{(n-1) ! r !}\right), \quad k(0, n)=0 .
$$

\section{СПИСОК ЛИТЕРАТУРЫ}

[1] Г. Фикера. Теоремы существования в теории упругости. М.: Мир, 1974. [2] В. А. Кондратьев, О.А. Олейник // УМН. 1988. Т. 43. №5. С. 55-98. [3] V. A. Kondratiev, O. A. Oleı̌nik // Rend. Mat. Appl. (7). 1990. V. 10. P. 641-666. [4] О. А. Матевосян // Диффференц. уравнения. 1998. Т. 34. №6. С. 806-811. [5] О. А. Матевосян // УМН. 2003. T. 58. № 2. C. 169-170. [6] A.Douglis, L. Nirenberg // Comm. Pure Appl. Math. 1955. V. 8. Р. 503-538. [7] С. Агмон, А. Дуглис, Л. Ниренберг. Оценки вблизи границы решений эллиптических уравнений в частных производных при общих граничных условиях. I. М.: ИЛ, 1962. [8] С. В. Morrey. Multiple Integrals in the Calculus of Variations. New York: Springer-Verlag, 1966. 